\title{
Letter \\ Gold Nanoparticle Conjugated Water Soluble Multiwall Carbon Nanotubes
}

\author{
Tamoghna Bhattacharyya \\ Center for Research in Nanoscience \& Nanotechnology, University of Calcutta, \\ Kolkata 700019, West Bengal, India; tbhattacharyya2010@gmail.com
}

Received: 8 July 2018; Accepted: 29 August 2018; Published: 1 September 2018

\begin{abstract}
The poor solubility and dispersibility of carbon nanotubes in both aqueous and organic solvents restrict their applications in biological science. Herein, a simple and cheap method for synthesizing and purification of water soluble multiwall carbon nanotubes from coal combustion fly ash and characterization of this water soluble multiwall carbon nanotubes after conjugation with gold nanoparticles has been described.
\end{abstract}

Keywords: multiwall carbon nanotubes; fly ash; green synthesis; water solubility; gold nanoparticles

\section{Introduction}

The discovery of buckminsterfullerene [1] by Smalley, Curl, and Kroto, which was aimed to understand the mechanism of long chain carbon molecules, revolutionized the carbon-based nanoscience. Later Iijima, discovered another new carbon allotrope named as carbon nanotube (CNT). [2] Afterwards, carbon based nanostructures [3] became one of the research topics in the field of nanoscience and nanotechnology. Numerous methods have been developed for the preparation of carbon nanotubes (CNTs) such as arc-discharge [1-5], laser ablation [6], high-pressure catalytic decomposition of carbon monoxide (HiPCO) [7], electrophoretic deposition (EPD) [8], flame synthesis [9], pyrolysis [10], chemical vapor deposition (CVD) [11], hot filament CVD [12], plasma enhanced CVD [13], radio-frequency [14], and microwave power sources [15]. However, for most of the strategies except a few require, high reaction temperature, complexity in processing and long synthesis time are always needed. Catalytic decomposition of hydrocarbons also requires a complex purification process to get rid of the metal catalyst particles [16]. Among all these synthetic routes for the synthesis of these carbon nanostructures, the chemical vapor deposition (CVD) route is mainly favored because of scalability and potentially low cost [17]. Although CNTs have unique mechanical, optical, electronic, and quantum mechanical properties, but the unfortunate aspect of CNTs is the poor solubility and dispersibility in both aqueous and organic solvents due its hydrophobic nature. It restricts their applications in the field of biological sciences. Herein, a simple and cheap method for synthesizing gold nanoparticles conjugated water soluble multi-walled carbon nanotubes (wsMWCNTs) has been described which may open up a new direction in biology, particularly in targeted drug delivery.

\section{Materials and Methods}

Coal combustion fly ash; solvents like toluene, acetonitrile, methanol, ethanol, pet-ether; and acetone were used. Analytical grade nitric acid was used for oxidative treatment.

\subsection{Synthesis and Soxhlet Purification of Raw Product from Fly Ash}

In this work, coal combustion fly ash, a bulk waste product, was used to produce multiwall carbon nanotubes (MWCNTs) $[18,19]$. Fly ash which was captured by electrostatic precipitators before the flue 
gases reach the chimneys was collected from a boiler from a local factory. First, fly ash was repeatedly washed with boiling toluene, acetonitrile, and alcohol respectively using soxhlet extraction technique. Then, the product was taken in a thimble and soxhlet extraction was carried out by pet-ether, followed by toluene and finally by acetonitrile. After three days, the extraction was judged to be complete as fluorescent orange colored soluble material ceased to leach out from the soxhlet thimble. The product obtained after soxhlet extraction was washed with methanol followed by water and finally vacuum dried. The entire process was repeated until about $5 \mathrm{gm}$ of the purified product was collected.

\subsection{Oxidative Treatment of Soxhlet Purified Product Using Concentrated $\mathrm{HNO}_{3}$}

About $5 \mathrm{gm}$ of the purified product was refluxed in an aqueous solution of cold nitric acid $(200 \mathrm{~mL})$ for $48 \mathrm{~h}$ [20]. A proportion of the product went into the solution. The un-dissolved residue was separated by centrifugation and the centrifugate was evaporated to dryness on a water bath to yield a black solid. Further purification of this black solid was done with boiling toluene, acetonitrile, alcohol, and distilled water respectively to yield about $1 \mathrm{gm}$ of water soluble multi-walled carbon nanotubes (wsMWCNTs).

\subsection{Synthesis of Gold Nanoparticle Modified wsMWCNTs}

$0.1 \mathrm{mg} / \mathrm{mL}$ wsMWCNTs was treated with $5 \mathrm{~mL}$ thiol in a bath sonicator for $30 \mathrm{~min}$ and refluxed at room temperature to yield thiol coated wsMWCNTs. Later gold nanoparticles with a size of $\sim 20 \mathrm{~nm}$ were treated with this thiol coated wsMWCNTs overnight to have gold nanoparticle conjugated wsMWCNTs.

\section{Results and Discussion}

\subsection{Elemental Analyses of wsMWCNTs}

The elemental compositions of wsMWCNTs were measured using a Thermo Scientific FLASH (Waltham, MA, USA) 2000 elemental analyzer from Thermo Scientific Fisher, USA (CHNS-O) based on combustion method. A series of elemental analyses indicated that the abundances of carbon and oxygen were found to be $42.2 \mathrm{wt} \%$ and $54.6 \mathrm{wt} \%$, respectively, in the product. The high abundance in oxygen in the product is due to the presence of oxygen functional groups (e.g., $-\mathrm{COOH},-\mathrm{OH}$ ).

\subsection{Structural Studies of wsMWCNT before and after GNP Conjugation Using Raman Spectroscopy}

The finger print of MWCNTs i.e., the Raman spectra (WITEC Alpha 300R confocal Raman system equipped with a frequency-doubled Nd: YAG laser (532 nm, $2.33 \mathrm{eV}$ ) and a 20× objective mounted on an Olympus optical microscope) of wsMWCNTs and gold nanoparticle conjugated wsMWCNTs are shown in Figure 1. The spectrum demonstrates the two prominent peaks the $G$ band, present at $\sim 1585 \mathrm{~cm}^{-1}$, is also known as the tangential band and arises from the E2g mode corresponds to the stretching mode in the graphite plane of the graphite plane and the second D-band, at $\sim 1353 \mathrm{~cm}^{-1}$ corresponds to disorder-induced stretching modes of graphite. Mainly attributed due to the presence of structural defects. The spectrum showed the high intensity of D-band corresponds to high order of derivatization during oxidative treatment. The spectrum after modification with GNPs shows clear evidence for the downshift of the all D-line $\left(1342 \mathrm{~cm}^{-1}\right)$ and G-line $\left(1602 \mathrm{~cm}^{-1}\right)$ which directly corresponds to the wreaking of C-C stretching frequency vibration due to extensive derivatization by thiols and conjugation with GNPs. 


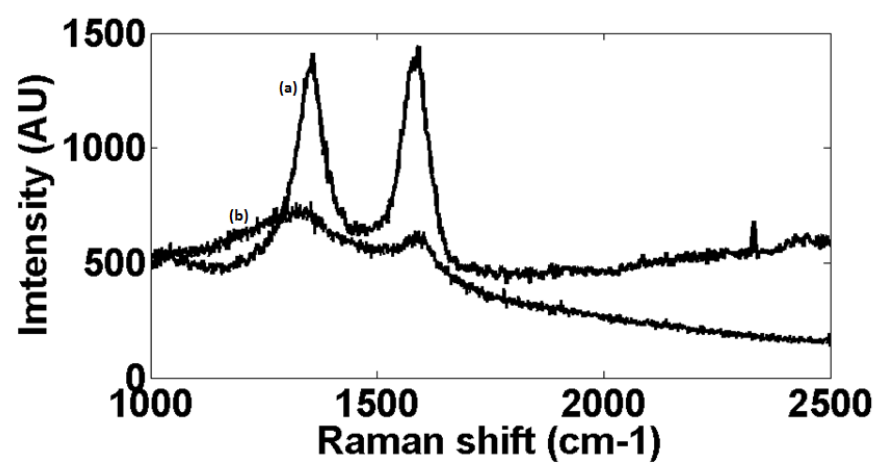

Figure 1. Raman spectra of (a) wsMWCNTs and (b) GNP conjugated wsMWCNTs.

The Raman spectra provide an important characteristic features of wsMWCNTs and wsMWCNTs conjugated with gold nanoparticles.

\subsection{TEM Investigation of wsMWCNT before and after GNP Conjugation}

The TEM images (JEOL JEM-2100 TEM system) as shown in Figure 2, give further information about the morphology and microstructure of wsMWCNTs before and after GNP conjugation. It is evident from the figure that GNPs are attached with thiol coated wsMWCNTs.
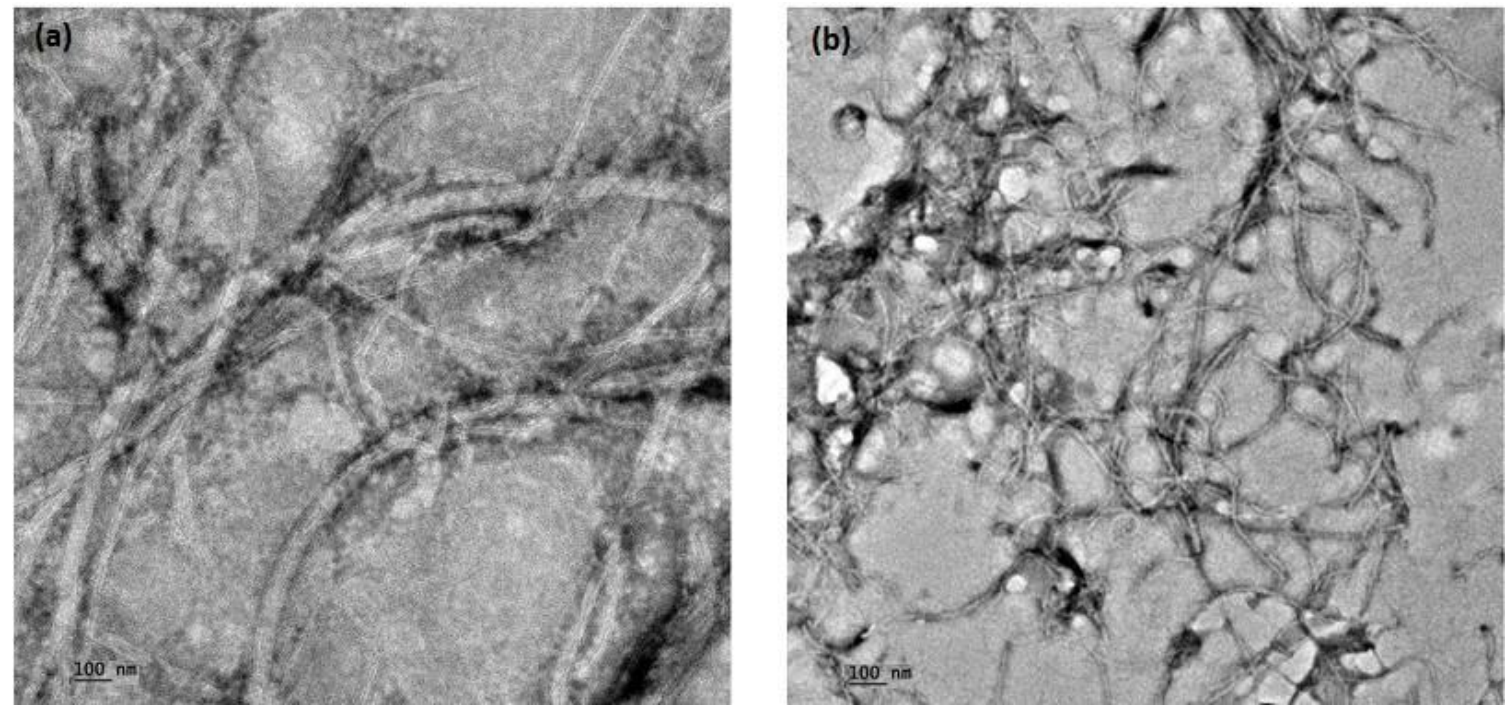

Figure 2. (a) TEM image of wsMWCNTs and (b) GNP conjugated wsMWCNTs.

\subsection{Absorption Spectroscopic Study of wsMWCNT before and after GNP Conjugation}

The absorption spectra (Agilent Cary 60 UV-vis spectrophotometer, Agilent, Santa Clara, CA, USA) clearly shows the plasmon of GNPs at $520 \mathrm{~nm}$ which is red shifted to $540 \mathrm{~nm}$ (as shown in Figure 3) after modification with wsMWCNTs while it is completely absent for wsMWCNTs. The red shift in plasmon is attributed to the interaction of GNPs with wsMWCNTs. 


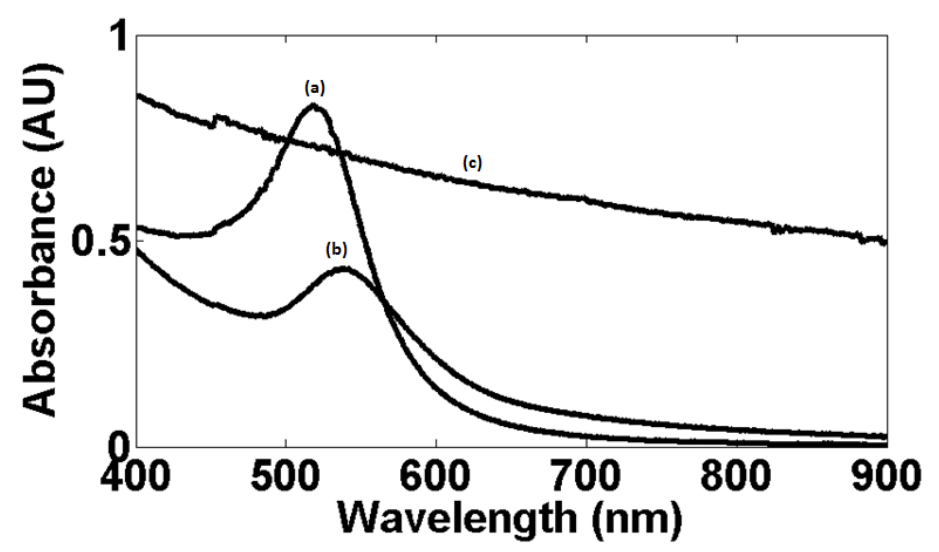

Figure 3. UV-vis spectra of (a) GNP (b) GNP conjugated wsMWCNTs and (c) wsMWCNTs.

\subsection{Fluorescence Study of wsMWCNT before and after GNP Conjugation}

The fluorescence spectrum (Nikon Eclipse Ti-U, Nikon Instruments, Melville, NY, USA) of wsMWCNTs shows a clear maximum at $430 \mathrm{~nm}$ which is due to the surface passivation of the MWCTs. The spectrum is red-shifted to $470 \mathrm{~nm}$ (as shown in Figure 4) after decorating with GNPs. Indirectly, the red-shift clearly carries the signature of gold nanoparticle conjugated wsMWCNTs.

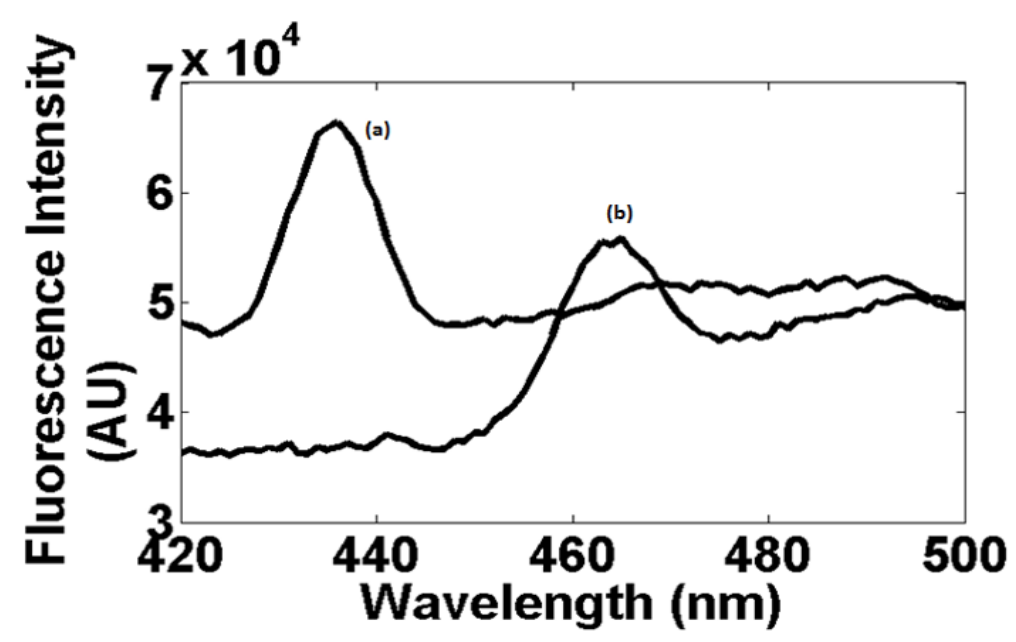

Figure 4. Fluorescence spectra of (a) wsMWCNTs and (b) GNP conjugated wsMWCNTs.

\subsection{Photo Co-Relation Spectroscopic Study of wsMWCNT before and after GNP Conjugation}

The average hydrodynamic radius (Malvern Nano-ZS, ZEN 3600, Malvern Panalytical, Malvern, UK) of GNPs suddenly increased to $\sim 110 \mathrm{~nm}$ which is $\sim 20 \mathrm{~nm}$ (see Figure 5) for GNP. 

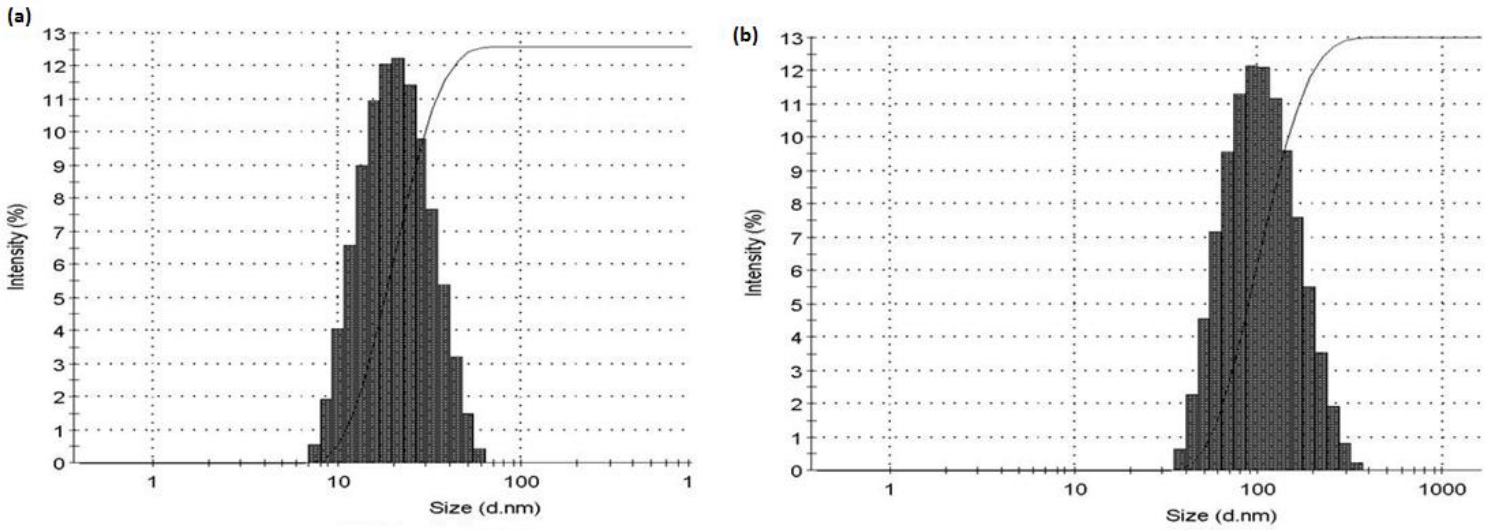

Figure 5. Average hydrodynamic radius of (a) GNP (b) GNP conjugated wsMWCNTs.

The increase in average size also proves that the GNPs are covalently attached with the wsMWCNTs to form a complex of wsMWCNTs modified with GNPs.

\section{Conclusions}

In summary, a simple and cheap method to synthesize and purify water soluble gold nanoparticle conjugated multi-wall carbon nanotubes from coal combustion fly ash has been described. It may open many future prospects to use these gold nanoparticle conjugated water soluble multiwall carbon nanotubes in the field of biological sciences, especially in targeted drug delivery.

Funding: This research received no external funding.

Conflicts of Interest: The authors declare no conflict of interest.

\section{References}

1. Kroto, H.W.; Heath, J.R.; O’Brien, S.C.; Curl, R.F.; Smalley, R.E. C60: Buckminsterfulleren. Nature 1985, 318, 162. [CrossRef]

2. Iijima, S. Helical microtubules of graphitic carbon. Nature 1991, 354, 56-58. [CrossRef]

3. Dresselhaus, M.S.; Dresselhaus, G.; Eklund, P.D. Science of Fullerenes and Carbon Nanotubes; Academic Press: San Diego, CA, USA, 1996.

4. Ugarte, D. Curling and closure of graphitic networks under electron-beam irradiation. Nature 1992, 359, 707. [CrossRef] [PubMed]

5. Ebbesen, T.W.; Ajayan, P.M. Large-scale synthesis of carbon nanotubes. Nature 1992, 358, 220. [CrossRef]

6. Eklund, P.C.; Pradhan, B.K.; Kim, U.J.; Xiong, Q.; Fischer, J.E.; Friedman, A.D.; Holloway, B.C.; Jordan, K.; Smith, K.M.W. Large-scale production of single-walled carbon nanotubes using ultrafast pulses from a free electron laser. Nano Lett. 2002, 2, 561. [CrossRef]

7. Nikolaev, P.; Bronikowski, M.J.; Bradley, R.K.; Rohmund, F.; Colbert, D.T.; Smith, K.A.; Smalley, R.E. Gas-phase catalytic growth of single-walled carbon nanotubes from carbon monoxide. Chem. Phys. Lett. 1999, 313, 91. [CrossRef]

8. Boccaccini, A.R.; Cho, J.; Roether, J.A.; Thomas, B.J.C.; Minay, E.J.; Shaffer, M.S.P. Electrophoretic deposition of carbon nanotubes. Carbon 2006, 44, 3149. [CrossRef]

9. Yuan, L.; Saito, K.; Pan, C.; Williams, F.A.; Gordon, A.S. Nanotubes from methane flames. Chem. Phys. Lett. 2001, 340, 237. [CrossRef]

10. Terrones, M.; Grobert, N.; Olivares, J.; Zhang, J.P.; Terrones, H.; Kordatos, K.; Hsu, W.K.; Hare, J.P.; Townsend, P.D.; Prassides, K.; et al. Controlled production of aligned-nanotube bundles. Nature 1997, 388, 52. [CrossRef]

11. Li, W.Z.; Xie, S.S.; Qian, L.X.; Chang, B.H.; Zou, B.S.; Zhou, W.Y.; Zhao, R.A.; Wang, G. Large-scale synthesis of aligned carbon nanotubes. Science 1996, 274, 1701-1703. [CrossRef] [PubMed] 
12. Ren, Z.F.; Huang, Z.P.; Xu, J.W.; Wang, W.J.; Bush, P.; Siegel, M.P.; Provencio, P.N. Synthesis of large arrays of well-aligned carbon nanotubes on glass. Science 1998, 282, 1105-1107. [CrossRef] [PubMed]

13. Merkulov, V.L.; Melechko, A.V.; Guillorm, M.A.; Lowndes, D.H.; Simpson, M.L. Alignment mechanism of carbon nanofibers produced by plasma-enhanced chemical-vapor deposition. Appl. Phys. Lett. 2001, 79, 2970-2972. [CrossRef]

14. Shiratori, Y.; Hiraoka, H.; Takeuchi, Y.; Itoh, S.; Yamamoto, M. One-step formation of aligned carbon nanotube field emitters at $400{ }^{\circ} \mathrm{C}$. Appl. Phys. Lett. 2003, 82, 2485-2487. [CrossRef]

15. Küttel, O.M.; Groening, O.; Emmenegger, C.; Schlapbach, L. Electron field emission from phase pure nanotube films grown in a methane/hydrogen plasma. Appl. Phys. Lett. 1998, 73, 2113-2115. [CrossRef]

16. Thess, A.; Lee, R.; Nikolaev, P.; Dai, H.J.; Petit, P.; Robert, J.; Xu, C.H.; Lee, H.Y.; Kim, S.G.; Colbert, D.T.; et al. Crystalline Ropes of Metallic Carbon Nanotubes. Science 1996, 273, 483-487. [CrossRef] [PubMed]

17. Hata, K.; Futaba, D.N.; Mizuno, K.; Namai, T.; Yumura, M.; Iijima, S. Water-assisted highly efficient synthesis of impurity-free single-walled carbon nanotubes. Science 2004, 306, 1362-1364. [CrossRef] [PubMed]

18. Dunens, O.M.; MacKenzie, K.J.; Harris, A.T. Synthesis of multiwalled carbon nanotubes on fly ash derived catalysts. Environ. Sci. Technol. 2009, 43, 7889-7894. [CrossRef] [PubMed]

19. Yasui, A.; Kamiya, Y.; Sugiyama, S.; Ono, S.; Noda, H.; Ichikawa, Y. Synthesis of carbon nanotubes on fly ashes by chemical vapor deposition processing. IEEJ Trans. Electr. Electron. Eng. 2009, 4, 787-789. [CrossRef]

20. Kim, K.H.; Jo, W.H. A strategy for enhancement of mechanical and electrical properties of polycarbonate/multi-walled carbon nanotube composites. Carbon 2009, 4, 1126-1134. [CrossRef]

(C) 2018 by the author. Licensee MDPI, Basel, Switzerland. This article is an open access article distributed under the terms and conditions of the Creative Commons Attribution (CC BY) license (http://creativecommons.org/licenses/by/4.0/). 